\title{
Identifcation and Location of Partial Discharge Defects in Medium Voltage AC Cables
}

\author{
Muhammad Shafiq ${ }^{1}$, Guillermo Robles ${ }^{2}$, Amjad Hussain $^{3}$, Kimmo Kauhaniemi ${ }^{1}$ \& Matti Lehtonen ${ }^{4}$ \\ ${ }^{1}$ School of Technology and Innovations, University of Vaasa, Vaasa, Finland \\ ${ }^{2}$ Department of Electrical Engineering, Universidad Carlos III de Madrid, Madrid, Spain \\ ${ }^{3}$ Department of Electrical and Computer, Engineering, American University of Kuwait, Kuwait \\ ${ }^{4}$ School of Electrical Engineering, Aalto University, Finland \\ Corresponding Author: muhammad.shafiq@uwasa.fi
}

\begin{abstract}
The presence of partial discharge (PD) in electrical equipment is the confirmation or indication of an ongoing degradation of its dielectric insulation. PD study is a widely used tool for condition monitoring of medium voltage (MV) cables. The location of the defects can be considered as one of the most important tasks of diagnosis in underground installations of MV cables. A lot of references can be found on the location of single PD sources along the cable. However, several PD defects can be active simultaneously along a cable section. This paper presents an experimental study on a MV cable having two PD sources and noise. Adopting one end measurement technique, a high frequency current transformer (HFCT) is used to capture the PD current pulses. A simple approach of identification of the PD signals originated from different PD sources is presented based on wave-shape study along with a time domain reflectometry analysis. The presented measurement methodology and time domain analysis technique provides a convenient way for detection and location of more than one PD sources along a cable section.
\end{abstract}

\section{Introduction}

Power distribution system consists of different types of lines carrying electric power from medium voltage (MV) substation to the consumers via distribution transformers. These lines are underground cables, overhead covered conductor (CC), and bare conductor overhead lines. Due to reduced failure rate, environmental conditions, esthetical preferences, and public safety of the cables, the overhead lines are being replaced by underground cables.

Insulation faults are the major cause of failure of MV cables. Such faults cause the initiation of partial discharges that can be monitored using appropriate sensors and can be located using suitable techniques. An increased interest has been shown in past decades to improve the available monitoring and diagnostic solution. A lot of work has been done to determine the detection and location of the PD faults [1]-[2]. Most of the work is done to detect and locate the presence of a single PD defect on a section of a cable. Recently progresses have been made to identify multiple faults using signals processing techniques, as well as to identify the type of the PD defect as corona, surface, internal, etc. [3]-[4]. Similarly, there has been recent progresses in the detection and location of multiple PD sources in the transformers using acoustic sensors based on the travelling time of the shock wave created by the PD [5]. However, the diagnostics of multiple PD faults that are active simultaneously have not been focused yet. Recently, reference [6] presents a work to detect the presence of the multiple PD sources along a cable section using power spectral separation. The use of such technique is valuable; however, its implementation needs high expertise on signal processing techniques, combined with artificial intelligence, some prior knowledge of PD defects, and assumptions of the number of PD sources, to guide the technique to make the signal identification. Therefore, along with the sophisticated techniques, it is required to keep developing the conventional technique based on simple studies that can provide simple and convenient ways to initiate the diagnostics.

This paper presents a simple approach of waveform analysis in the time domain to identify the presence of more than one PD source along a cable section that are active simultaneously. The work is based on experimental investigations carried out in a laboratory environment. Once the PD signals are identified and separated, the time domain reflectometry technique is used to localize the PD signals.

The paper is organized as follows; section 2 presents the understanding of localizing breakdown sections across the defective part of a cable insulation. Section 3 describes the characteristic parameters of a PD current pulse that can help in the fault diagnostics. Section 4 presents a brief discussion about the behavior of cables as medium of propagation of the PD current pulse and the measurement system to acquire them. Experimental investigation of the PD defects, fault identification, and their location is done in Section 5, while Section 6 concludes the findings.

\section{Partial discharge in the MV cables}

Cavities, cracks, air bubbles and treeing in the dielectric insulation cause deterioration of the cable and grow with time and in extent due to various stresses (thermal, electrical, ambient and mechanical stresses, also called TEAM stresses). At a certain time, insulation strength becomes so weak that suffers a complete breakdown.

The physical model of the cavity emerging inside the insulation of a MV cable and its surrounding healthy insulation is shown in Fig. 1(a). Assuming that the solid insulation of the cable has a thickness $h_{\mathrm{i}}$ and dielectric permittivity $\varepsilon_{\mathrm{i}}$, while $h_{\mathrm{c}}$ is the thickness of disc shaped cavity with a dielectric permittivity $\varepsilon_{\mathrm{c}}$. Due to different dielectric constants of the insulation between the electrodes, different capacitances emerge which 
proportionally divides the voltage applied across the electrodes. To analyze the PD ignition, a well-known electrical (a-b-c) model of the cavity inside insulation is used and shown in Fig. 1(b). Here $C_{\mathrm{ia}}, C_{\mathrm{ib}}$, and $C_{\mathrm{ic}}$ are the capacitances of the respective sections. The voltage $V_{\mathrm{a}}$ across $C_{\mathrm{ia}}$ is essentially divided across $C_{\mathrm{ib}}$ and $C_{\mathrm{ic}}$ which represents the faulty region. The voltage $V_{\mathrm{c}}$ across $C_{\mathrm{ic}}$ is of major concern for PD generation and is expressed as,

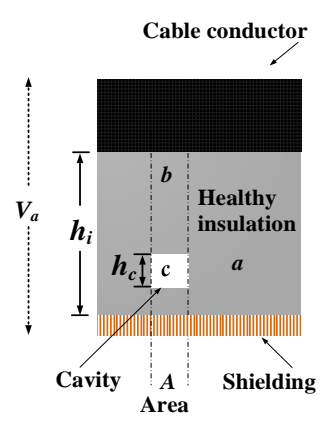

(a)

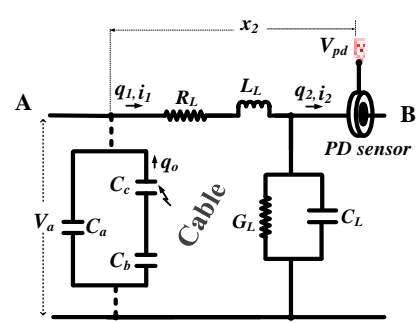

(b)
Figure 1. (a) Physical model of the cavity inside insulation (upper half of the cross-sectional view of cable) and (b) electrical a-b-c model of the cavity inside cable insulation.

$V_{\mathrm{c}}=V_{\mathrm{a}} \frac{C_{\mathrm{ib}}}{C_{\mathrm{ib}}+C_{\mathrm{ic}}}$.

In terms of respective dielectric permittivity, it can be expressed as,

$V_{\mathrm{c}}=V_{\mathrm{a}} \frac{1}{1+\frac{\varepsilon_{\mathrm{c}}}{\varepsilon_{\mathrm{i}}}\left(\frac{h_{\mathrm{i}}}{h_{\mathrm{c}}}-1\right)}$.

The electric field $E_{\mathrm{a}}$ due to supply voltage $V_{\mathrm{a}}$ is uniformly distributed across insulation between the conductors. Based on $V_{\mathrm{a}}=E_{\mathrm{a}} d$, where $E_{\mathrm{a}}$ is the electric field due to potential $V_{\mathrm{a}}$ across the electrodes, the electric field $E_{\mathrm{c}}$ across the cavity can be described as

$E_{\mathrm{c}}=E_{\mathrm{a}}\left(\frac{h_{\mathrm{i}}}{h_{\mathrm{c}}}\right) \frac{1}{1+\frac{\varepsilon_{\mathrm{c}}}{\varepsilon_{\mathrm{i}}}\left(\frac{h_{\mathrm{i}}}{h_{\mathrm{c}}}-1\right)}$.

Considering the practical behavior of a partial breakdown of insulation within the particular cavity, if the size of the cavity and relative permittivity are considerably smaller than the solid insulation, as will usually be the case, the $E_{\mathrm{c}}$ will be significantly greater than $E_{\mathrm{a}}$.

Assuming $h_{\mathrm{c}}=0.6 \mathrm{~mm}$ inside a $12 / 20 \mathrm{kV}$ cross-linked polyethylene (XLPE) cable having $6.6 \mathrm{~mm}$ in thickness $\left(h_{\mathrm{i}}\right)$ insulation. For $\varepsilon_{\mathrm{c}}=1$ (relative permittivity) and $\varepsilon_{\mathrm{i}}=2.3$ (for XLPE), the electric field intensity in the cavity is approximately 2.3 times that of the surrounding insulation at a certain applied voltage. The dielectric breakdown strength of XLPE is $E_{\mathrm{BA}}=21$ $\mathrm{kV} / \mathrm{mm}$ (approximately) whereas for the air-cavity is $E_{\mathrm{BC}}=3$ $\mathrm{kV} / \mathrm{mm}$ (approximately). Therefore, for the above-described cavity, the applied voltage $V_{\mathrm{BC}}$ at which the breakdown starts to occur can be determined in terms of $E_{\mathrm{BC}}$ and dimensional arrangement of the cavity as in,

$V_{\mathrm{BC}}=E_{\mathrm{BC}}\left(1+\frac{\varepsilon_{\mathrm{c}}}{\varepsilon_{\mathrm{i}}}\left(\frac{h_{\mathrm{i}}}{h_{\mathrm{c}}}-1\right)\right) h_{\mathrm{c}}$.

When the voltage across the cavity rises to $\mathrm{V}_{\mathrm{BC}}$, a discharge takes place within the capacitive cavity which is called PD inception voltage and it is calculated as $V_{\mathrm{BC}}=9.6 \mathrm{kV}$ in this case, even when the nominal phase-to-ground voltage is $12 \mathrm{kV}$ for the $12 / 20 \mathrm{kV}$ cable under test. Once the process is triggered, the insulating materials start to deteriorate progressively and eventually lead to a complete breakdown.

\section{Characteristics of a PD current pulse}

A PD current pulse can be expressed as

$i_{\text {pulse }}(t)=A\left(e^{-\alpha_{2} t}-e^{-\alpha_{1} t}\right)$

A typical PD pulse is compared with the practically measured PD pulse in Fig. 2 where $A$ is the peak value of the pulse, $\alpha_{l}$ is the rate of rise-time and $\alpha_{2}$ is the rate of fall-time.

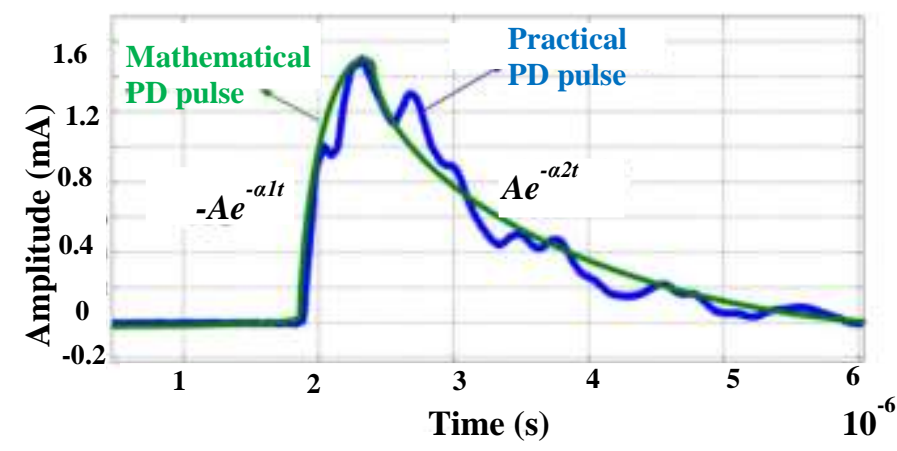

Figure 2. Typical PD pulse in electrical power systems, mathematical (ideal) pulse in green and practically measured PD pulse in blue.

Rise-time of the pulse is an important parameter of PD activity and also determines the highest frequency components of the PD signal. The faster the rise-time, the higher will be the frequency of the PD pulse. The rise-time of PD pulse depends on the drift velocity and the size of the cavity (the path travelled by the discharged avalanche). For a gas-filled cavity, the drift velocity $v_{d}$ in $\mathrm{cm} / \mathrm{s}$ can be determined theoretically as [7].

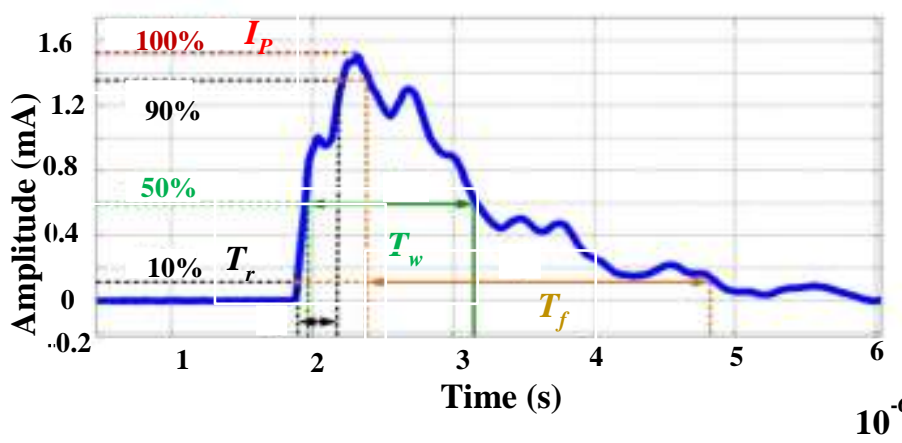

Figure 3. Characteristics parameters of a PD pulse. 
$v_{\mathrm{d}}=1.334 .10^{6}+4.22 \cdot 10^{5}\left(\frac{E_{\mathrm{ci}}}{p}\right)$

where $E_{c i}(\mathrm{kV} / \mathrm{cm})$ is the electric field intensity at PD inception and $p$ is the atmospheric pressure of the cavity. Hence the risetime (transit time) $T_{r}$ is calculated as

$T_{\mathrm{r}}=\frac{h_{\mathrm{c}}}{v_{\mathrm{d}}}$

where $h_{c}$ is the depth of the cavity (void) which is the distance that the charges travel during a discharge event. Equation (7) presents an estimation of the rise-time, however local factors such as temperature and conductivity of the cavity also have an impact [8]. For a cavity of $0.2 \mathrm{~cm}$ in diameter in two polyethylene films with a void depth of $0.08 \mathrm{~mm}$ and $0.12 \mathrm{~mm}$, the rise-time $\left(T_{r}\right)$ of PD pulse (shown in Fig. 3 ) is calculated as $0.754 \mathrm{~ns}$ and $0.991 \mathrm{~ns}$ respectively [7]. The fall- time $T_{f}$ of the induced PD pulse depends upon the impedance of the cable, propagation characteristics of the medium (cable system) a given pulse must travel through prior to be measured, and the impedance of the measuring system [9]. The above mentioned parameters of the PD pulse vary for different types of insulation in electrical components. Size and type of insulation defect also affects the value of these parameters.

The shape of the pulse and its characteristics play an important role during the analysis of the ongoing PD activity. In order to measure a PD signal accurately, the measuring sensor should be designed with compatible sensitivity and bandwidth. The amplitude of the PD pulse sets the intended sensitivity of the sensor. Similarly, rise-time, fall-time, and pulse width which refer to the frequency contents of a pulse signal, provide an idea for selection of a suitable bandwidth of the sensor. Based on practical experiences, the amplitude of PD pulses lies within the range up to few milli-amperes while the pulse width ranges between nanoseconds to microseconds.

\section{Possible effects of MV cable and measurement system-Discussion}

PD current pulses are generated and travel along the line or cable and then they are sensed by the sensors. Figure 4 shows the operational diagram of the PD measurement setup in which a PD pulse $P_{1}$ is induced on the line and travels through the line inheriting the characteristics of the line parameters and captured by the sensor transducer; finally, the measured signal is observed at the output of sensor interface through a data acquisition system.

Here $i_{1}(t)$ is the primary or original signal emerged at the PD site, $i_{2}(t)$ is the PD signal at the measuring point having travelled through the cable, and $i_{3}(t)$ is the received signal, while $T_{c}$ and $T_{s}$ are the transfer functions of the cable and the sensors respectively, that incorporate the effects of the characteristics of the cable and the sensor systems.

It has been studied that the cables have significant effects on the propagating PD signals considering two aspects; frequency domain and time domain. Because of the specific geometric construction of conductors, dielectric insulation, and semiconducting layers, the electrical parameters of the cable such as resistance, inductance and capacitance, affects the PD signals propagating along the cables. Attenuation and dispersion are two major effects that high frequency pulses have to bear during their propagation. The attenuation constant represents the metal losses proportional to the resistivity; and the dielectric losses due to the loss tangent, the conductivity of the dielectric, and the stray radiation. The in-depth analysis presented in [10] demonstrates that there exists a severe attenuation for the PD signals of higher frequencies (above $30 \mathrm{MHz}$ ) in MV cables. The dispersion exhibits the velocity depends of the frequency components of the PD signals while travelling along the line. This dependence leads to the spreading of the measured PD pulse. The dispersion is mainly due to the high frequency behavior of the semiconducting layer. High frequency components of the PD signal face immense attenuation. Consequently, the signal bandwidth is reduced and the original PD shape is spread in the time domain [11]-[12].

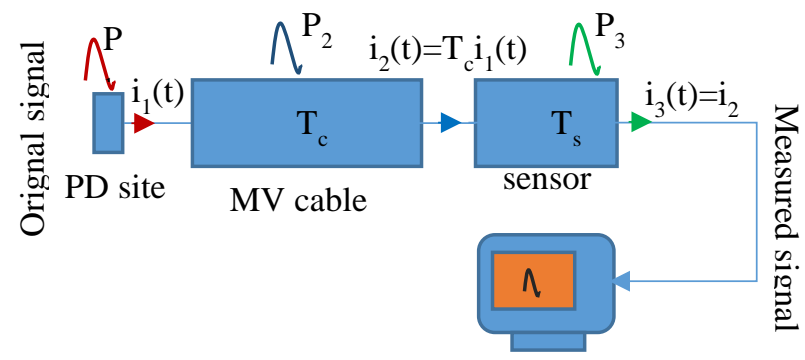

Data acquisition system

Figure 4. Layout of the PD measurement process along the MV cable.

Sensors are designed in such a way that the effect posed by their circuitry and any internal signal processing should be removed by signal conditioning, compensation, and calibration. Considering electromagnetic induction sensors designed in [13][14], the major effects that appears during measurement process are, mutual inductance, the $2^{\text {nd }}$ order RLC (resistanceinductance-capacitance) response, and time derivative of the input current. These effects have been compensated and as an outcome, the original signal is produced. Therefore, it can be stated that an ideal sensor the effect of sensor's characteristics should not appear at the output and therefore a sensor's output is a copy of the input signal.

\section{Two PD faults on the MV cable}

\subsection{Experimental setup}

Experimental investigations were done on a $20-\mathrm{kV}$ shielded MV cable with a low-density high-molecular-weight polyethylene insulation. The length of the cable was $l=150 \mathrm{~m}$, while the conductor section was AWG 18 or $1 \mathrm{~mm}^{2}$ and the external jacket had a diameter of $7.7 \mathrm{~mm}$.

Two PD faults were created at different locations $P_{l}$ and $P_{2}$. It was a new cable that had no PD faults. The ends of the cables were peeled off to get the shieldings separated in order to install 
the PD sensor. The separated shielding at End $A$ was acting as PD source $P_{1}$ while at the End $B$ the shielding was immersed in the transformer oil to avoid the PDs. Similar kind of arrangement was made to have a PD source in the middle of the cable section. The cable was split into two sections (Figure 5 and 6), and connected again to join the inner conductors and the shielding with crocodile clips. This creates an intentional defect in the electrical insulation between conductor and shielding at point $\mathrm{P}_{2}$. PD source at $\mathrm{P}_{1}$ was at the End $A$ of the cable and therefore located at distance $x_{1}=0 \mathrm{~m}$ from End $A$. The distance of $P_{2}$ from End $A\left(x_{2}=84.5 \mathrm{~m}\right)$ was selected randomly at a considerable distance from the cable ends.

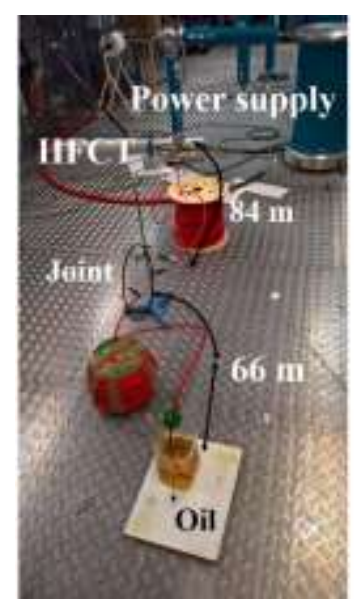

Figure 5. Laboratory setup for PD measurements.

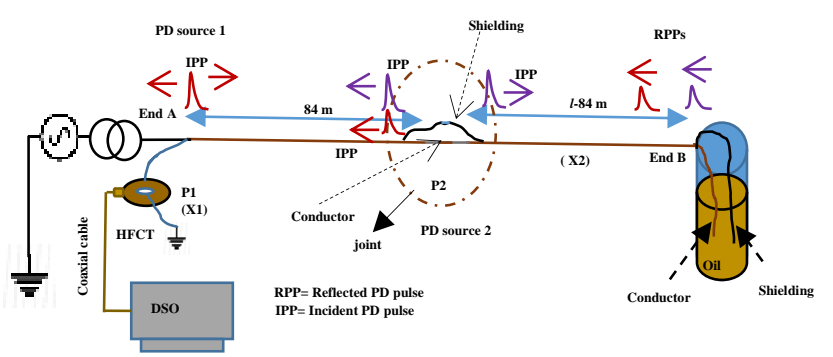

Figure 6. Layout the PD measurement setup.

A MV transformer was used with variable control to adjust the applied voltage that energized the cable. The applied voltage was raised up to $4 \mathrm{kV}$ and the $\mathrm{PD}$ signals were measured at the End A using an HFCT. The trigger of the oscilloscope was set low in order to capture the possible low PDs. The data was acquired at a sampling rate of $1 \mathrm{GS} / \mathrm{s}$. As the PD emission is a continuous activity with a large number of pulses emitted from each PD source after every certain interval, therefore 500 signals were recorded with each window consisting of 5000 samples. The starting of each captured window is based on the trigger level of the PD pulse and it lasted for 5000 samples. The length of the number of samples (5000) or the duration $(5 \mu \mathrm{s})$ of the window was selected by keeping in view to record enough time for each incident PD pulse measured by the HFCT and its two $\left(1^{\text {st }}\right.$ and $2^{\text {nd }}$ ) reflections reaching to the HFCT by travelling 300 meters that requires $2 \mu$ s or 2000 samples. The third reflection was too attenuated to be of interest and therefore it has not been taken into account.

Figure 7 presents the data of 500 signals in the same data file. For detailed analysis, the data was divided into sets of 10 individual signals to observe the shape of the signals and their reflections as shown in Fig.8 while more clear analysis can be seen in Fig. 9.

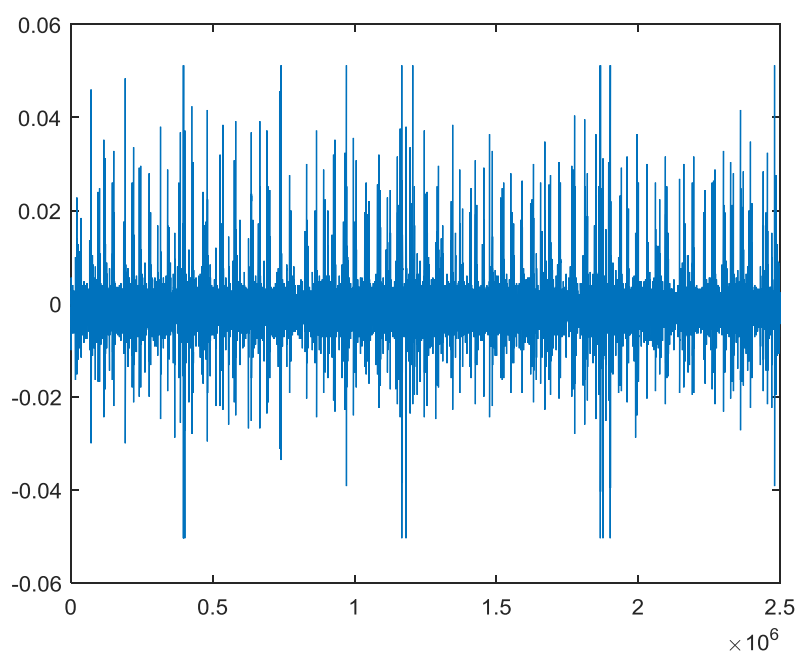

Figure 7. PD data of 500 signals.

Observing Signal 1 to Signal 10, it can be seen that the pulses have different amplitudes, polarity, wave shape, and pulse repetition rate. The difference between the amplitude and the polarity is because of the phase location of the pulses. Due to sinusoidal supply, the amplitude of the pulses is greater at the higher instantaneous voltage and the polarity is opposite due to positive and negative cycle of the supply voltage. The wave shape depends upon the shape, size, or type of the PD source. From the analysis of the data, it was also observed that the number of pulses during certain time from the PD 1 source are greater than the number of pulses captured from the PD 2 type. Based on the dimension of the PD cavity or defect type the time taken for the charging and discharging of the capacitance across the cavity varies that leads to the generation of the PD pulses at different rate even for the same applied voltage.

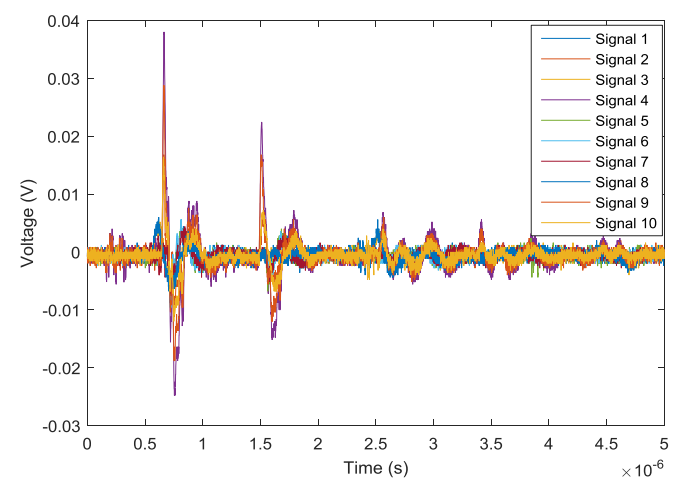

Figure. 8. PD analysis in the sets of 10 consecutive PD signals. 
The detection of the different types of PDs on the cable can be initiated by observing the wave-shape of the first pulse of each signal. Signal 3 and Signal 8 have the similar wave shapes while rest of the signals have the same wave shapes. Therefore, a perception can be made that two types of PDs are present; Signal 1 and its alike can be considered from PD1 source and the Signal 3 and its alike are considered from PD2 source. Extending the analysis by observing the reflections of each signal can provide a better understanding. It can be clearly seen that the reflected pulses of both types of signals have different times of arrival that confirms that these signals are emerging from the sources that are at different locations along the cable.

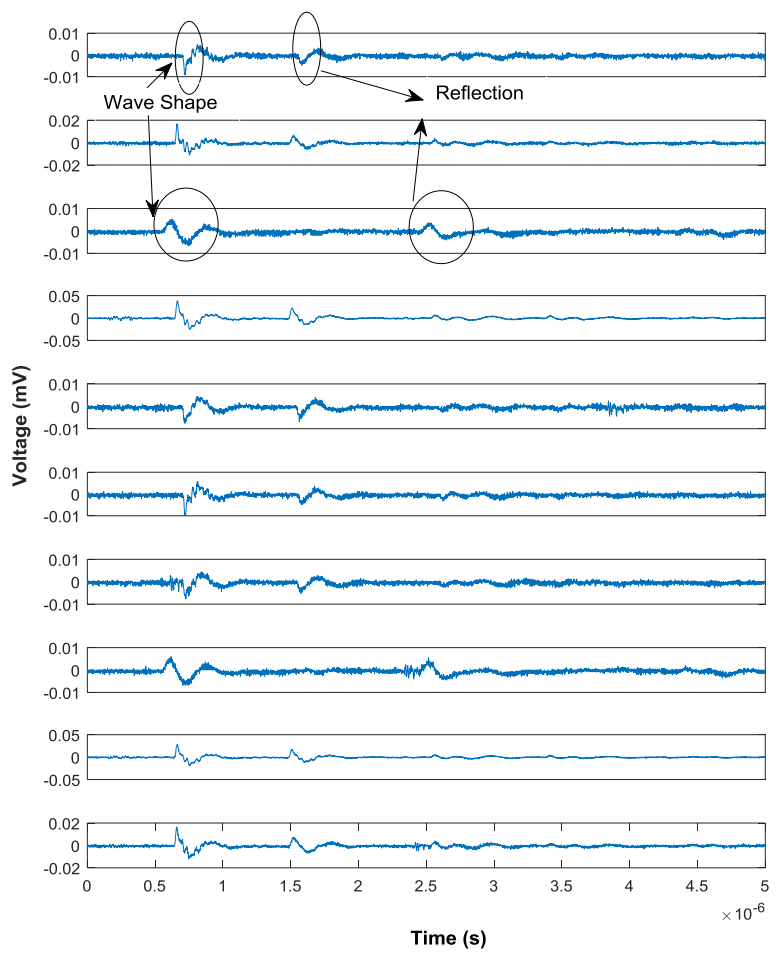

Figure 9. PD Analysis in time domain to observe wave-shape and time of reflections.

\subsection{TDR based analysis for fault location}

Time domain reflectometry determines the location of the source based on the wave propagation velocity of cable and time difference of arrival between the original pulse and its reflection arriving at the measuring point. The wave velocity of the cable has been determined experimentally as $1.575 \times 10^{8} \mathrm{~m} / \mathrm{s}$.

Assuming a cable of length $l$, the PD pulse generated from a point $\mathrm{P}$ in the middle starts to travel in one direction toward end A to be captured by the sensor at time $t_{l}$ after traveling a distance $x$. At the same time, the PD pulse starts traveling from point $\mathrm{P}$ in the opposite direction toward end $\mathrm{B}$ of the cable by covering first the distance $l-x$, and then, it is reflected back to reach the sensor at End $A$ after a time $t_{2}$. The distance of the fault from End $A$ can be calculated

$$
x=l-\frac{\Delta t v}{2}
$$

Where $\Delta t=t_{2}-t_{1}$.

The two signals identified as emerging from different PD sources are shown in Fig. 11. The time of the PD pulse can be taken as the time of start or the first peak of the PD pulse. In Figure 11(a), the first pulse of the signal PD1 arrives at time $t_{1}=$ $0.662 \mu \mathrm{s}$ while the reflected pulse arrives at time $t_{R 1}=1.512 \mu \mathrm{s}$ therefore $\Delta t_{1}=0.850 \mu$ s can be used in equation 8 .

$x_{1}=l-\frac{\Delta t_{1} v}{2}=0.85 \mathrm{~m}$

Similarly, considering the first pulse of the signal PD2 arrives at time $t_{2}=0.629 \mu \mathrm{s}$ while the reflected pulse arrives at time $t_{R 2}=2.526 \mu \mathrm{s}$ therefore having $\Delta t_{2}=1.90 \mu \mathrm{s}$ can be used in equation 8.

$x_{2}=l-\frac{\Delta t_{2} v}{2}=83.27 \mathrm{~m}$

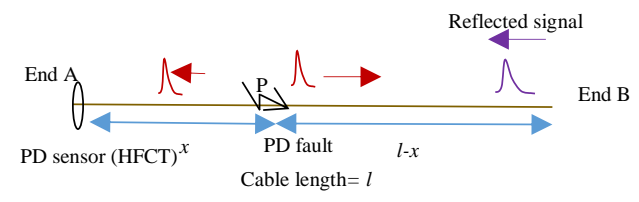

(a)

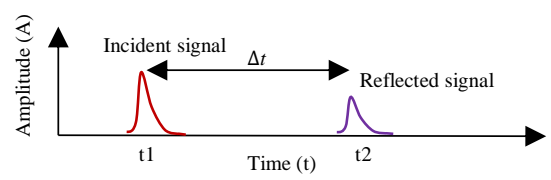

(b)

Figure 10. (a). The propagation of the PD signals away from the PD site, (b). Time difference of arrival of the original and reflected PD signals.
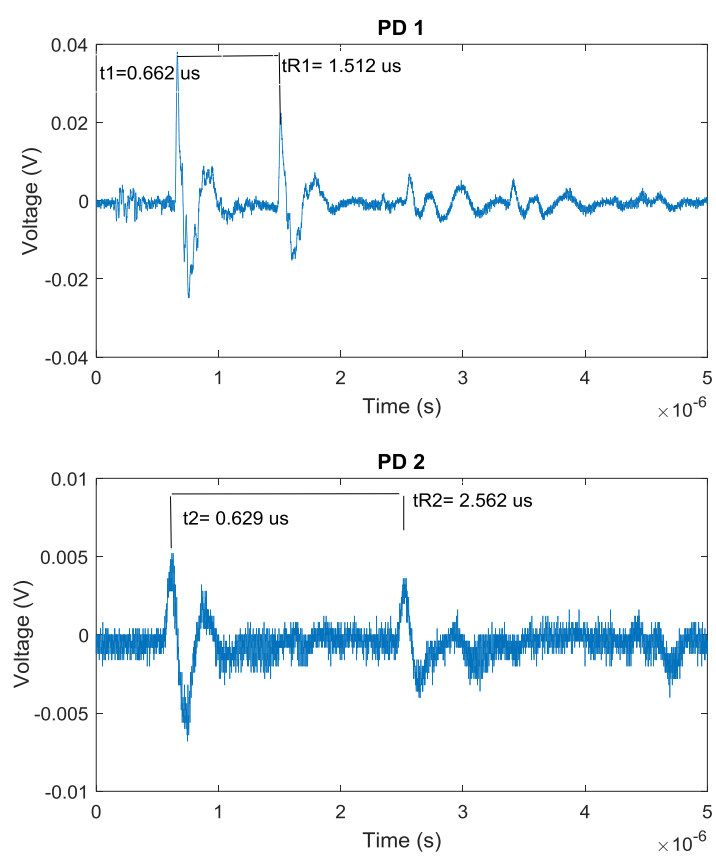

Figure 11. TDR based location of the two PD sources, (a). Time difference of arrival for PD 1 type, (b). Time difference of arrival for PD 2 type. 
Considering these measurements, PD source 1 and 2 are located at distances $0.85 \mathrm{~m}$ and $83.27 \mathrm{~m}$ respectively while the actual distances are $0 m$ and $84 m$ respectively that represents a small error. The sampling period is a source of the error the sampling period can cause minor errors in a systemic manner. In this work, the measurements were recorded at a sampling period of $1 \mathrm{GS} / \mathrm{s}$. This means the measurement are recorded with a resolution of $0.15 \mathrm{~m}$. Therefore, there is always a possibility of the error of $0.15 \mathrm{~m}$ distance during extraction of the results from the measurement waveforms. The issue of sampling period can affect the accuracy of the location diagnostics during measurement of time difference or arrival. Although the distance of the defect points from the cable end has been measured carefully, however as the cable was unrolled from a drum, some bending may cause an error in its measurement.

The XLPE has emerged as a robust dielectric insulation that makes the insulation of a cable sections quite reliable. However, joints are always needed and are the most vulnerable components of an MV cable. When the PD measurements are performed at a certain part of the cable feeder having number of joints, there is a possibility that more than one joints are suffering with the PD defects simultaneously. In this case, the presented methodology will be useful to identify and locate the faulty joints efficiently. This scenario is more likely in the paper insulated cable that are installed decades ago and have increased number of joints due to repairs during their life time.

\section{Conlusions}

PD current pulses emerging from the defect locations propagate along the cable and can be captured by the sensors at the joint or cable ends. The cables and sensors have their own properties that are incorporated into the propagating PD pulses. Although the PD signals are emerged from the same type of cable, at the same operational stresses and measured by the same sensor, based on the type or shape of the defect, the emerged PD signals are different from each other and they maintain their shapes and signatures. Based on the shape and the time of the arrival of the reflected pulses, identification of the sources can be made if they are located at different locations. The presented method can be used for identification of the PD sources based on visual analysis of the data in time domain and its outcomes can be valuable to compare or for dual verification of the algorithm based 'automated' ways of signal classification.

\section{Acknowledgement}

Authors are grateful to Prof. J. M. Martínez-Tarifa for his support in carrying out the experimental investigation at Universidad Carlos III de Madrid. This work is done under the project Smart Condition Monitoring of Power Grid that is funded by Academy of Finland (Grant No. 309412).

\section{References}

[1] Reid, A.J., Zhou, C., Hepburn, D.M., Judd, M.D., Siew, W.H. and Withers, P., "Fault location and diagnosis in a medium voltage EPR power cable".
IEEE Transactions on Dielectrics and Electrical Insulation, Vol. 20, Issue. 1, 2013, pp.10-18.

[2] Van der Wielen, P. C. J. M. "On-line detection and location of partial discharges in medium-voltage power cables." $\mathrm{PhD}$ Thesis, Eindhoven University of Technology Electrical Power Systems (EPS) Group, 2005.

[3] Hao, L., P. L. Lewin, and S. G. Swingler. "Identification of multiple partial discharge sources." in IEEE International Conference on Condition Monitoring and Diagnosis, 2008, pp. 118-121.

[4] Boya, Carlos, et al. "Identification of multiple partial discharge sources using acoustic emission technique and blind source separation." IEEE Transactions on Dielectrics and Electrical Insulation, Vol. 22, Issue. 3 2015, pp. 1663-1673.

[5] N. N. Ali, , J. A. Hunter, P. Rapisarda, and P. L. Lewin, 'Identification of multiple partial discharge sources in high voltage transformer windings. In 2014 IEEE Conference on Electrical Insulation and Dielectric Phenomena (CEIDP) (pp. 188-191). IEEE.

[6] G. Robles, M. Shafiq, and J. M. Martínez-Tarifa. "Multiple Partial Discharge Source Localization in Power Cables Through Power Spectral Separation and Time-Domain Reflectometry." IEEE Transactions on Instrumentation and Measurement, 2019.

[7] B. Ramachandra and R. S. Nema, "Characterization of Partial Discharge Pulses in Artificial Voids in Polyp Films used in Capacitors", in IEEE International Symposium on Electrical Insulation, Montreal, Canada, Jun. 1996.

[8] T. Seghier, D. Mahi, T. Lebey, and D. Malec, "The Effect of Space Charge on Partial Discharges Inception Voltage in Air Gaps Within High Density Polyethylene" Courrier Du Savoir - N¹0, Université Mohamed Khider Biskra, Algériepp, 2010, pp. 35-41.

[9] M. Fenger, "Sensitivity Assessment Procedure for Partial Discharge Measurements on Transmission Class Cables", in IEEE International Symposium on Electrical Insulation (ISEI 2008), Toronto, Canada, 2008, pp. 667-671.

[10] Clark, D., Mackinlay, R., Giussani, R., Renforth, L., \& Shuttleworth, R. Partial discharge pulse propagation, localisation and measurements in medium voltage power cables. In $48^{\text {th }}$ International Universities' Power Engineering Conference (UPEC), 2013, pp. 1-6.

[11] Shafiq, M., Kauhaniemi, K., Robles, G., Hussain, G. A., \& Kumpulainen, L. (2018). Partial discharge signal propagation in medium voltage branched cable feeder. IEEE Electrical Insulation Magazine, 34(6), 18-29.

[12] M. Tozzi "Partial discharge in power distribution electrical systems: Pulse propagation models and detection optimization," $\mathrm{PhD}$ dissertation, Dept. Electr. Eng., Univ. Bologna, Bologna, Italy, 2010.

[13] M. Shafiq, K. Kauhaniemi, G. Robles, M. Isa, and L. Kumpulainen, Online condition monitoring of MV cable feeders using Rogowski coil sensors for PD measurements. Electric Power Systems Research, 2019, 167, 150-162.

[14] Shafiq, Muhammad. "Design and implementation of partial discharge measurement sensors for on-line condition assessment of power distribution system components." Aalto University, Doctoral Dissertation, 2014. 